\title{
Dementia among Older Persons in Jamaica: Prevalence and Policy Implications
}

\author{
D Eldemire-Shearer ${ }^{1}$, K James ${ }^{2}$, P Johnson ${ }^{3}$, R Gibson², D Willie-Tyndale ${ }^{1}$
}

\begin{abstract}
Objective: To determine the prevalence of dementia and dementia types in Jamaica.

Methods: An embedded case-control design was used to investigate dementia within the ageing population. Cases (Mini-Mental State Examination [MMSE] scores of $\leq 20$ ) and controls (MMSE scores of $>20)$ were evaluated using DSM-IV protocol and magnetic resonance imaging. Prevalences (crude and age-adjusted) were calculated and distribution of dementia by type described.

Results: Dementia prevalence was 5.9\%. Alzheimer's pattern dementia accounted for $61.8 \%$ and vascular dementia 32.4\%. However, vascular disease was prominent in $45.5 \%$ of the Alzheimer's cases. Female gender and increasing age were associated with higher rates of dementia. Dementia was 38 times more likely in participants with MMSE scores below 20.

Conclusion: This first nationally representative study indicated that dementia rates in Jamaica were comparable with regional and global estimates. Regardless of the dementia type, vascular change was pervasive and suggested that synergistic efforts should be made to address underlying contributory factors. Cardiovascular and cerebrovascular risk reduction should be deliberately pursued as integral adjuncts to dementia risk reduction.
\end{abstract}

Keywords: Ageing, Alzheimer's disease, dementia, prevalence

\section{La demencia entre las personas de edad en Jamaica: prevalencia e implicaciones para la implementación de políticas}

D Eldemire-Shearer ${ }^{1}, \mathrm{~K}_{\text {James}}{ }^{2}$, P Johnson ${ }^{3}$, R Gibson ${ }^{2}$, D Willie-Tyndale ${ }^{1}$

\begin{abstract}
RESUMEN
Objetivo: Determinar la prevalencia de los tipos de demencia y demencia en Jamaica.

Métodos: Se utilizó un diseño de caso-control incrustado para investigar la demencia dentro de la población en proceso de envejecimiento. Los casos (puntuación $\leq 20$ en el Mini Examen del Estado Mental [MEEM]) y los controles (puntuación > 20 en el MEEM) fueron evaluados usando el protocolo DSM-IV y la imagen por resonancia magnética. Se calcularon prevalencias (crudas y ajustadas por edad) y se describió la distribución de la demencia por tipo.

Resultados: La prevalencia de demencia fue de 5.9\%. El Alzheimer representó el $61.8 \%$ y la demencia vascular 32.4\%. Sin embargo, la enfermedad vascular fue prominente en el $45.5 \%$ de los casos de Alzheimer. El género femenino y la edad creciente se asociaron con tasas más
\end{abstract}

From: ${ }^{1}$ Mona Ageing and Wellness Centre, The University of the West Indies, Mona, Jamaica, West Indies, ${ }^{2}$ Department of Community Health and Psychiatry, The University of the West Indies, Mona, Jamaica, West Indies and ${ }^{3}$ Department of Surgery, Radiology, Anaesthesia and Intensive Care, The University of the West Indies, Mona, Jamaica, West Indies.
Correspondence: Professor D Eldemire-Shearer, Mona Ageing and Wellness Centre, The University of the West Indies, Mona, Kingston 7, Jamaica, West Indies. Email: denise.eldemireshearer@uwimona. edu.jm 
altas de demencia. La demencia fue 38 veces más probable en los participantes con puntuaciones de MEEM por debajo de 20.

Conclusión: Este primer estudio nacionalmente representativo indicó que las tasas de demencia en Jamaica eran comparables con los estimados regionales y globales. Independientemente del tipo de demencia, el cambio vascular fue generalizado y sugirió que se hicieran esfuerzos sinérgicos para abordar los factores contribuyentes subyacentes. Debe buscarse deliberadamente la reducción del riesgo cardiovascular y cerebrovascular como adjuntos integrantes de la reducción del riesgo de demencia.

Palabras clave: Envejecimiento, enfermedad de Alzheimer, demencia, prevalencia

\section{West Indian Med J 2018; 67 (1): 2}

\section{INTRODUCTION}

Dementia is a clinical syndrome of progressive impairment of memory and intellectual function, including executive function, language and motor skills, resulting in significant impairment of cognition, function and behaviour as well as an increase in care needs. There is evidence that the prevalence of dementia is increasing particularly in low- and middle-income countries such as Jamaica and Trinidad and Tobago $(1,2)$. The 2015 estimated worldwide prevalence was $5-7 \%$, but Latin America and the Caribbean had a higher prevalence of $7.1-8.5 \%$, while Africa was lower at $2-4 \%(1,3-6)$. For the population aged 60 years or over, Alzheimer's Disease International estimated the 2015 crude prevalence specifically for the Caribbean at $6.5 \%$ and that relative to 2015 , proportionate increases of $60 \%$ and $183 \%$ would be realized by 2030 and 2050, respectively, for dementia [all types] (4). Consequently, among the Jamaican elderly, approximately 300000 (7), the current estimated 19000 persons with dementia will increase to 31000 in 2030 and 55000 in 2050.

There are several dementias. The two main types are Alzheimer's disease and vascular dementia. There is also mixed dementia in which there is evidence of Alzheimer's and vascular disease, specifically cerebrovascular disease. Other types include Lewy Body (4, 6). The prevalence of each type varies by region. While Alzheimer's is most common, averaging $60 \%$ of cases, vascular dementia varies from $15 \%$ of the cases in Latin America and the Caribbean to 20\% in Europe and 50\% in Japan (8). Increasingly, studies have noted the importance of vascular change in Alzheimer's $(9,10)$. Studies have also highlighted the potential to reduce dementia prevalence by modifying vascular factors $(9,10)$.

Diagnosis of dementia type, care and support are associated with a high social and economic cost both to the country and to the individual. In actuality, dementia is the largest single contributor to the need for care by older persons $(4,6)$. This begs the question: how ready is the Caribbean and specifically Jamaica to deal with both the increased cost (including social, health and long-term care costs) and efforts to reduce the number of cases? Important to answering this question is local and regional information, and this study aimed to determine the prevalence of dementia and dementia types in Jamaica.

\section{SUBJECTS AND METHODS}

A nationally representative survey of 2943 persons aged 60 years or above was conducted in 2012, using a twostage cluster sample with a probability proportional to size sampling strategy. Details of the methodological approach and procedures have been published elsewhere $(11,12)$. From the cohort of 2943, Mini-Mental State Examination (MMSE) scores were available for 2782 persons, and it was decided to investigate the cohort further with regard to the pattern and distribution of various types of dementia (including Alzheimer's and vascular dementia), as well as associated clinical and radiological (magnetic resonance imaging (MRI)) features. Recent demographic analysis from the United States of America (13) suggested, among older persons, a prevalence of about $11 \%$ for Alzheimer's disease, the major type of dementia. Given the objectives of the present study, bearing the latter figure in mind and along with the usual 95\% confidence level and 5\% margin of error, the minimum required sample size would be 280 (visit www. raosoft.com $\backslash$ samplesize.html for the online sample size calculator used).

For efficiency, an embedded case-control study was constituted. Cases and controls were determined on the basis of MMSE scores. Of the 2782 persons with MMSE scores, 2073 had scores greater than 20 and 709 
had scores 20 or below. From the participants with available MMSE scores, a random selection of 170 persons with scores of 20 or below was made. These constituted the targeted cases for inclusion in the study. Similarly, a random selection of 170 persons with scores greater than 20 (controls) was also done which constituted the targeted controls for inclusion in the study. The planned ratio of cases to controls was approximately $1: 1$.

The cut point of 20 was chosen based on the available literature (14-19). Providing guidance on the interpretation of MMSE scores, Folstein et al explicitly ascribed increased odds of dementia to scores below 21 (20). The sensitivity, specificity, positive and negative predictive values $(83.7 \%, 64.3 \%, 86.7 \%, 50.0 \%$, respectively) of a cut point of 20 have been documented by Pezzotti et $a l$, allowing for estimates of anticipated proportions of cognitive impairment and dementia among cases and controls, predictive values $-86.7 \%$ and $50 \%$ (21). Based on the latter figures and the sample numbers described, the study had more than $90 \%$ power to detect statistically significant differences in cognitive impairment or dementia prevalence between cases and controls (visit www.obg.cuhk.edu.hk/ResearchSupport/StatTools/ SSiz2Props_Pgm.php for the online power/sample size calculator used).

For the diagnosis of dementia, DSM-IV criteria were used, and a semi-structured interview was carried out in the community. Analysis of the agreement between field staff diagnoses and those of the consultant psychiatrist (gold standard) showed a kappa value of approximately $0.9(p<0.001)$ which, based on Cohen-Mansfield et al (22) and McHugh (23), confirmed significant and almost perfect agreement and inter-rater reliability.

Clinical and radiological features of dementia cases were also described. There was some attrition of selected subjects: 21 (the result of death), 6 (refusals) and 12 (migration). The final number in the sample was 301 (158 cases and 143 controls). The MMSE was repeated for all participants in the case-control study to reflect the MMSE score closest to the time of the MRI examinations.

Informed consent was obtained from each patient or person appointed as power of attorney for enrolment in the study as well as for the MRI and clinical diagnosis of dementia procedures. The 301 participants in the study were asked to undergo MRI scans and DSM-IV diagnostic testing for dementia as well as depression screens.

Data were entered into a database and analysed with the help of SPSS software, version 20. $P$-values below 0.05 were deemed statistically significant. Ethical approval was obtained from The University of the West Indies, Mona, Ethics Committee.

\section{RESULTS}

\section{Participants by age and gender}

A total of 301 subjects were involved in this case-control study on dementia. Cases (MMSE scores of 20 or below) numbered 158 and comprised $52.5 \%$ of the sample while the remaining 143 persons or $47.5 \%$ were controls (MMSE scores greater than 20). Table 1 shows the distribution of cases and controls by age.

Table 1: Distribution of study participants by age, gender and MMSE score category

\begin{tabular}{|c|c|c|c|c|c|}
\hline & & $\begin{array}{c}\text { MMSE > } 20 \\
(\text { (n (\%)) }\end{array}$ & $\begin{array}{c}\text { MMSE } \leq 20 \\
(\mathrm{n}(\%))\end{array}$ & $\begin{array}{c}\text { Total } \\
(\mathrm{n}(\%))\end{array}$ & $p$-value \\
\hline \multicolumn{6}{|c|}{ Age (years) } \\
\hline \multirow[t]{4}{*}{$60-69$} & & & & & 0.858 \\
\hline & Male & $38(57.6)$ & $28(42.4)$ & $66(100)$ & \\
\hline & Female & $38(60.3)$ & $25(39.7)$ & $63(100)$ & \\
\hline & Total & $76(58.9)$ & $53(41.1)$ & $129(100)$ & \\
\hline \multirow[t]{4}{*}{$70-79$} & & & & & 0.453 \\
\hline & Male & $24(53.3)$ & $21(46.7)$ & $45(100)$ & \\
\hline & Female & $33(45.8)$ & $39(54.2)$ & $72(100)$ & \\
\hline & Total & $57(48.7)$ & $60(51.3)$ & $117(100)$ & \\
\hline \multirow[t]{4}{*}{$\geq 80$} & & & & & $0.054 *$ \\
\hline & Male & $6(35.3)$ & $11(64.7)$ & $17(100)$ & \\
\hline & Female & $4(10.5)$ & 34 (89.5) & $38(100)$ & \\
\hline & Total & $10(18.2)$ & 45 (81.8) & $55(100)$ & \\
\hline \multirow[t]{4}{*}{ All ages } & & & & & 0.103 \\
\hline & Male & $68(53.1)$ & $60(46.9)$ & $128(100)$ & \\
\hline & Female & 75 (43.4) & 98 (56.6) & $173(100)$ & \\
\hline & Total & $143(47.5)$ & $158(52.5)$ & $301(100)$ & \\
\hline
\end{tabular}

$p$-values based on Chi-square statistics except * where Fisher's Exact Test was used.

Of the 301 study participants, 129 (42.9\%) were aged 60-69 years, $38.9 \% 70-79$ years and $18.3 \% 80$ years or over. Age was associated with MMSE score category. As age increased, persons were more likely to have MMSE scores of 20 or below $\left(\chi^{2}(1)=25.77, p<0.0011\right)$. Among those aged 80 years or over, $81.8 \%$ were in the MMSE score category of 20 or below.

As illustrated in Table 1, the majority (57.5\%) were female (173 of 301), as expected from the national population figures. There were increasing proportions of females as age increased: these proportions being $48.8 \%$ (63 of 129), 61.5\% (72 of 117) and 69.1\% (38 of 55) for the age groups of 60-69 years, 70-79 years and 80 years or over, respectively. The association between age and 
gender was statistically significant $\left(\chi^{2}(1)=7.77, p=\right.$ 0.021 ). There was no statistically significant relationship between gender and MMSE category. Among males in the study, $46.9 \%$ had MMSE scores of 20 or below while among females the corresponding figure was $56.6 \%$. Table 1 highlights distributions of gender with MMSE categories layered by age group to control for age as a confounding factor.

\section{Dementia}

Application of DSM-IV criteria identified 34 (11.4\%) study participants as having dementia (Table 2). Among controls (those with MMSE scores greater than 20), only one participant $(0.7 \%)$ was diagnosed with dementia. Comparatively, among cases (those with MMSE scores of 20 or below), $33(21.2 \%)$ had dementia. Persons with MMSE scores of 20 or below were almost 38 times more likely to have dementia than those with MMSE scores greater than 20. Of the 34 persons with dementia, $97 \%$ had MMSE scores of 20 or below. The only person with a score greater than 20 who was clinically diagnosed with dementia had a score of 21. Proportions of persons with dementia increased significantly with age: up from $3.9 \%$ to $10.3 \%$ to $30.9 \%$ among the age groups of $60-69$ years, $70-79$ years and 80 years or over, respectively (Table 3 ). Overall dementia rates among the genders were $7.9 \%$ for males and $14.0 \%$ for females. However, the difference was not statistically significant $(p=0.107)$.

Analysis within age groups showed a trend of higher rates of dementia among women except in the age group of 60-69 years (Fig. 1). In the youngest age cohort (60-69 years), rates were $4.7 \%$ and $3.2 \%$ for males and females, respectively. The corresponding rates for the age groups of 80 years or over were $17.6 \%$ and $36.8 \%$. Although there was a tendency for greater differences in the gender-specific rates of dementia with increasing age, statistical significance was not achieved: $p$-values based on Fisher's Exact Test being 1.00, 0.764 and 0.213 for the age groups of 60-69 years, 70-79 years and 80 years or over, respectively.

\section{Magnetic resonance imaging classification}

Magnetic resonance imagings were done for 300 persons $(99.7 \%$ of the study participants). No features suggestive of dementia were found in 266 study participants. The remaining 34 participants $(11.3 \%)$ had findings indicative of dementia, a 100\% concordance with the clinical diagnosis. The dominant pattern of dementia among these 34 subjects is displayed in Fig. 2. Alzheimer's pattern dementia (61.8\%) and vascular dementia (32.4\%) were the main types detected. There was one case of Lewy Body type of dementia (2.9\%).

Table 2: Frequency of dementia among cases and controls

\begin{tabular}{|c|c|c|c|c|c|}
\hline & \multicolumn{2}{|c|}{ Dementia diagnosis* } & \multirow{2}{*}{$\begin{array}{c}\text { Total } \\
(\mathrm{n}(\%))\end{array}$} & \multirow[t]{2}{*}{ Dementia rate } & \multirow{2}{*}{$\begin{array}{l}\text { Unadjusted odds ratio } \\
\text { (95\% confidence interval) }\end{array}$} \\
\hline & $\begin{array}{c}\text { Yes } \\
(\mathrm{n}(\%)) \\
\end{array}$ & $\begin{array}{c}\text { No } \\
(\mathrm{n}(\%)) \\
\end{array}$ & & & \\
\hline Cases (MMSE $\leq 20)$ & $33(21.2)$ & $123(78.8)$ & $156(100)$ & $0.212(21.2 \%)$ & $37.8(5,281)$ \\
\hline Controls (MMSE > 20) & $1(0.7)$ & $141(99.3)$ & $142(100)$ & $0.007(0.7 \%)$ & 1.0 (Reference) \\
\hline Total & $34(47.5)$ & $264(52.5)$ & $298(100)$ & & \\
\hline
\end{tabular}

* Dementia diagnosis was not available for three persons (two cases and one control did not complete the study).

Table 3: Dementia diagnosis by age and gender

\begin{tabular}{lrrrrr}
\hline & & \multicolumn{2}{c}{ Dementia diagnosis } & Total & p-value* \\
\cline { 3 - 5 } & & $\begin{array}{c}\text { Yes } \\
(\mathbf{n}(\mathbf{\%}))\end{array}$ & $\begin{array}{c}\text { No } \\
(\mathbf{n}(\mathbf{\%}))\end{array}$ & & \\
\hline Age (years) & & & & & $<0.001$ \\
& $60-69$ & $5(3.9)$ & $122(96.1)$ & $127(100)$ & \\
& $70-79$ & $12(10.3)$ & $104(89.7)$ & $116(100)$ & \\
$\geq 80$ & $17(30.9)$ & $38(69.1)$ & $55(100)$ & \\
Gender & All ages & $34(11.4)$ & $264(88.6)$ & $298(100)$ & \\
& & & & & 0.107 \\
& Male & $10(7.9)$ & $116(92.1)$ & $126(100)$ & \\
& Female & $24(14.0)$ & $148(86.0)$ & $172(100)$ & \\
\hline
\end{tabular}

* $p$-values based on Chi-square statistics

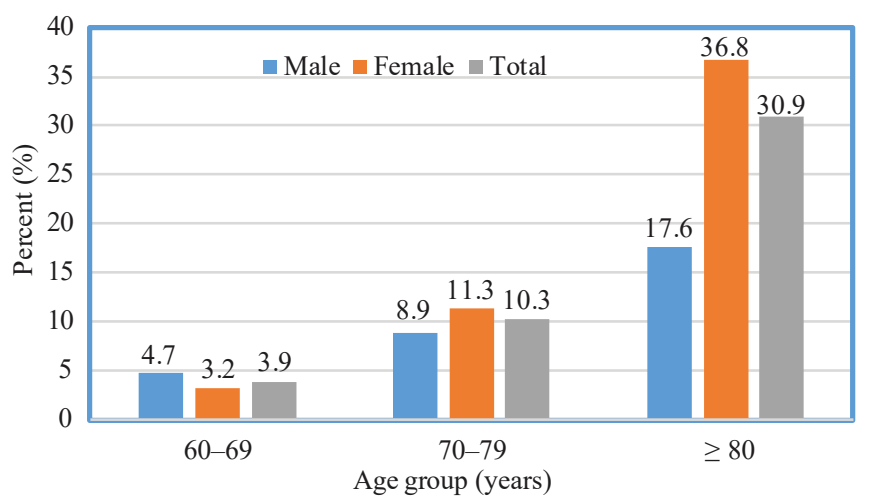

Fig. 1: Dementia rates by gender within age cohorts. 


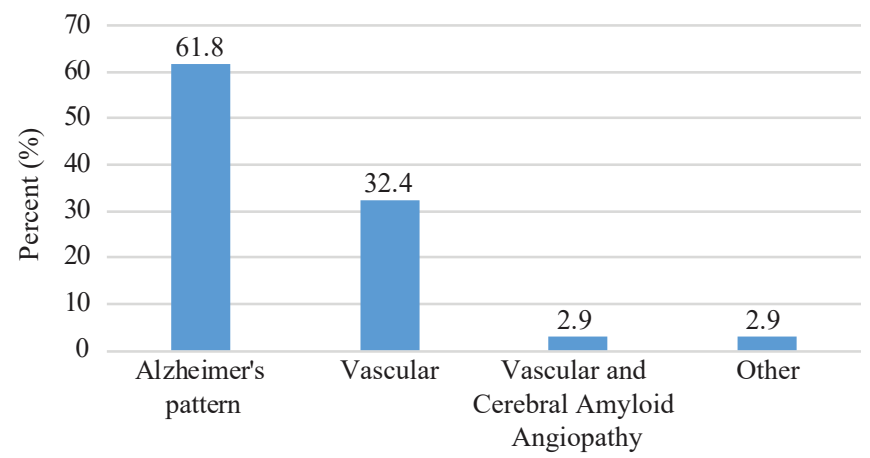

Fig. 2: Distribution of dominant dementia pattern/type $(n=34)$.

The Fazekas scale which quantifies the white matter hyper-intense lesions in brain MRI usually attributed to chronic small vessel disease was used, and $83.3 \%$ of those diagnosed with vascular dementia had the maximum level, ie Fazekas 3 (Table 4). Notably, 45.5\% of those with an Alzheimer pattern also had vascular changes (Fazekas 3).

All the 21 subjects with predominantly Alzheimer's pattern disease on MRI had MMSE scores of 20 or below. All were also clinically diagnosed as having dementia using DSM-IV criteria. Among the 11 persons with predominantly vascular type pattern, $90.9 \%$ had MMSE scores of 20 or below. All 11 with predominantly vascular type pattern on MRI were clinically diagnosed as having dementia using DSM-IV criteria. For those categorized on MRI as 'Vascular and Cerebral Amyloid Angiopathy' and 'Other', all had MMSE scores of 20 or below, and all were identified as having dementia using DSM-IV criteria.

\section{Prevalence of dementia}

The prevalence of dementia was derived from the observed rates of dementia among the categories of MMSE scores in the subsample of persons in the casecontrol study. Recognizing the relative proportions of the MMSE categories (MMSE scores $\leq 20$ and MMSE scores $>20)$ in the original larger sample used for the study of older persons by Eldemire et al (12), rates were

Table 4: Dementia pattern/type and Fazekas score category

\begin{tabular}{|c|c|c|c|c|c|}
\hline & $\begin{array}{c}\text { Alzheimer's } \\
\text { and other } \\
(\mathrm{n}(\%))\end{array}$ & $\begin{array}{l}\text { Vascular } \\
\text { (n (\%)) }\end{array}$ & $\begin{array}{c}\text { Total } \\
(\mathrm{n}(\%))\end{array}$ & $\begin{array}{c}\text { Fisher's } \\
\text { Exact } \\
\text { Test } \\
(p \text {-value) }\end{array}$ & $\begin{array}{c}\chi^{2}-\text { for } \\
\text { trend } \\
(p \text {-value })\end{array}$ \\
\hline \multicolumn{4}{|c|}{ Fazekas score category } & 0.066 & 0.035 \\
\hline 1 or 2 & $12(54.5)$ & $2(16.7)$ & $14(41.2)$ & & \\
\hline 3 & $10(45.5)$ & $10(83.3)$ & $20(58.8)$ & & \\
\hline Total & $22(100)$ & $12(100)$ & $34(100)$ & & \\
\hline
\end{tabular}

applied to generate the cases in the larger sample for the MMSE categories. The total cases derived were then used to calculate the prevalence of dementia in the original sample and were then extrapolated to the national population, the original study sample being already shown to be nationally representative (12).

In the study by Eldemire et al (12) which involved 2943 subjects, data on MMSE scores were available for 2782 persons ( $~ 95 \%$ of participants). The distribution of the number of older persons with MMSE scores $\leq 20$ and MMSE scores $>20$ is shown in Table 5. Applying the relevant category-specific dementia rates yielded the anticipated number of cases of dementia for each group. The total number of cases was thus 165 among the 2782 subjects, yielding a prevalence of 5.9\%.

For comparison of rates, with other studies in Jamaica and Western Europe, age-adjusted or age-standardized rates were computed. Age-adjusted rates are generated to account for underlying differences in the age distribution of populations over time or different locations since age composition by itself can affect overall rates of dementia. An earlier small community-based study from Jamaica reported a crude prevalence of $5.0 \%$, and the age-adjusted rate was $5.32 \%$, standardized to the Western European population (24). Using rates from the same Western Europe standard population described in the World Alzheimer Report 2009 (25), the age-adjusted rate for the current study was $6.06 \%$ (Table 6).

Table 5: Expected cases by MMSE category among participants in a 2012 study of older persons in Jamaica*

\begin{tabular}{lrrc}
\hline \multicolumn{1}{c}{ Category** } & Number & Dementia rate & $\begin{array}{c}\text { Expected cases } \\
\text { (rate x number) }\end{array}$ \\
\hline MMSE $\leq 20$ & 709 & $0.212(21.2 \%)$ & 150 \\
MMSE $>20$ & 2073 & $0.007(0.7 \%)$ & 15 \\
Total & 2782 & & 165 \\
\hline
\end{tabular}

* Eldemire-Shearer et al (12).

** MMSE scores not available for 161 persons.

Table 6: Comparison of age-standardized dementia prevalence by source

\begin{tabular}{|c|c|}
\hline Source & $\begin{array}{l}\text { Age-standardized prevalence } \\
(\%(95 \% \text { confidence interval }))\end{array}$ \\
\hline $\begin{array}{l}\text { World Alzheimer Report 2009* } \\
\text { (Western Europe population) }\end{array}$ & $6.92($ Reference $)$ \\
\hline $\begin{array}{l}\text { A small community study in } \\
\text { Jamaica** }\end{array}$ & $5.32(2.42,8.20)$ \\
\hline Current study & $6.06(5.18,6.96)$ \\
\hline
\end{tabular}

* Alzheimer's Disease International (25).

** Neita et al (24).

Indirect method of standardization was used, and Western Europe population was deemed the standard or reference population. 


\section{Cognitive changes}

Approximately $16 \%$ of the study participants had memory impairment (Table 7). Among those with dementia, all had memory impairment, consistent with this being a required condition for diagnosis. Comparatively, only $5.3 \%$ of those without dementia had memory impairment. Aphasia was noted in $7 \%$ of the participants. Among those with dementia, 52.9\% had aphasia, in contrast with $1.1 \%$ among those without dementia. Only $2.3 \%$ of the study participants had apraxia, and the rates were $20.6 \%$ among those with dementia and $0.0 \%$ among those without dementia. With regard to agnosia, $3.0 \%$ of the participants had this cognitive deficit. Rates among those with dementia and those without were $26.5 \%$ and $0.0 \%$ respectively. Almost $10 \%$ of the study participants had disturbances in executive function: $79.4 \%$ of persons with dementia had such disturbance compared to $0.8 \%$ among those without dementia. $P$-values confirmed the association between dementia and these cognitive deficits. Among those with dementia, $47.1 \%$ had two cognitive deficits while $52.9 \%$ had three or more. Of those diagnosed with

Table 7: Cognitive deficits and dementia diagnosis

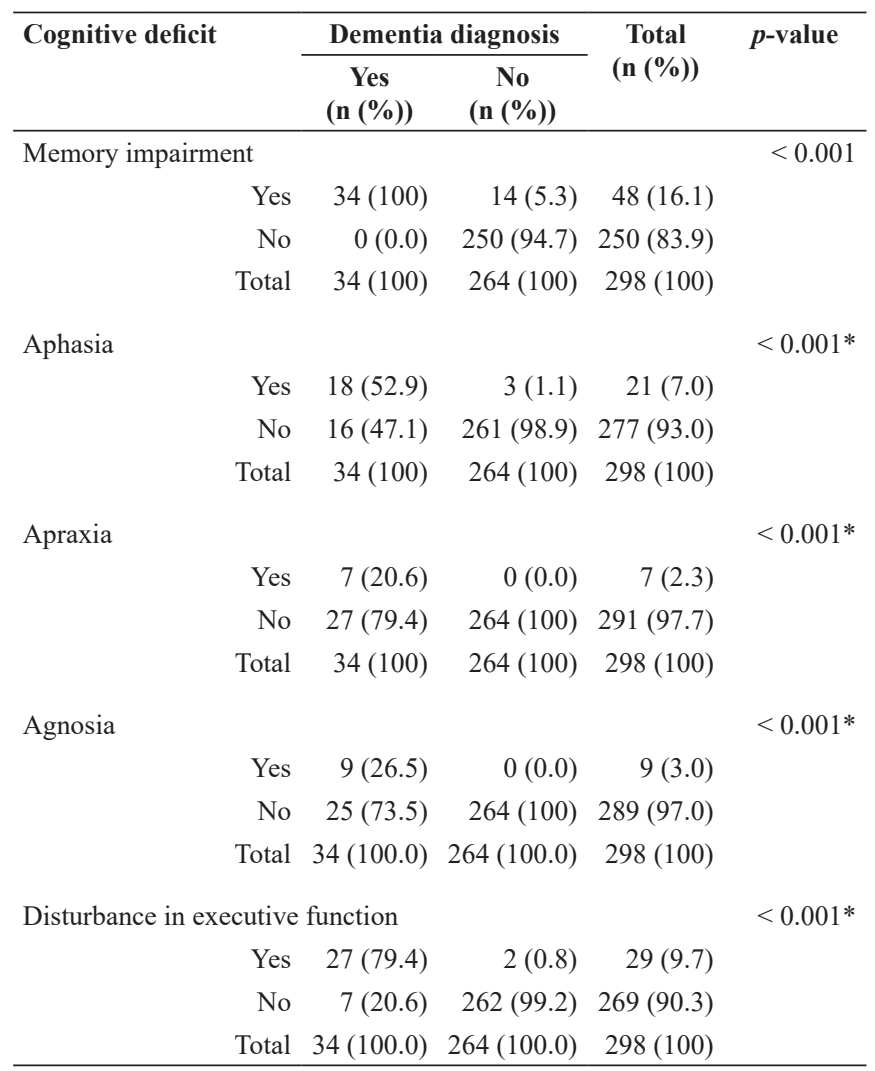

$p$-values based on Chi-square statistics except * where Fisher's Exact Test was used. dementia, 52.9\% had aphasia, reflecting communication difficulties related to production, expression and comprehension of language.

\section{Dementia and chronic disease}

Among persons diagnosed with dementia, 69.7\% had doctor-diagnosed hypertension while among those without dementia, $63.2 \%$ had doctor-diagnosed hypertension (Table 8). The difference was not statistically significant. Rates for diabetes were statistically similar: $30.3 \%$ among persons with dementia and $27.8 \%$ among those without.

An examination of the dementia pattern/type among persons identified with dementia with regard to their hypertension/diabetes status revealed that among those with predominantly Alzheimer's pattern, $28.6 \%$ had both hypertension and diabetes (Table 9). The corresponding figure for those with predominantly the vascular pattern was $16.7 \%$. The majority $(66.7 \%)$ of those with predominantly vascular-type dementia had hypertension, as did the majority $(71.5 \%)$ of those with predominantly Alzheimer's pattern. Although there was variation in

Table 8: Dementia and hypertension/diabetes

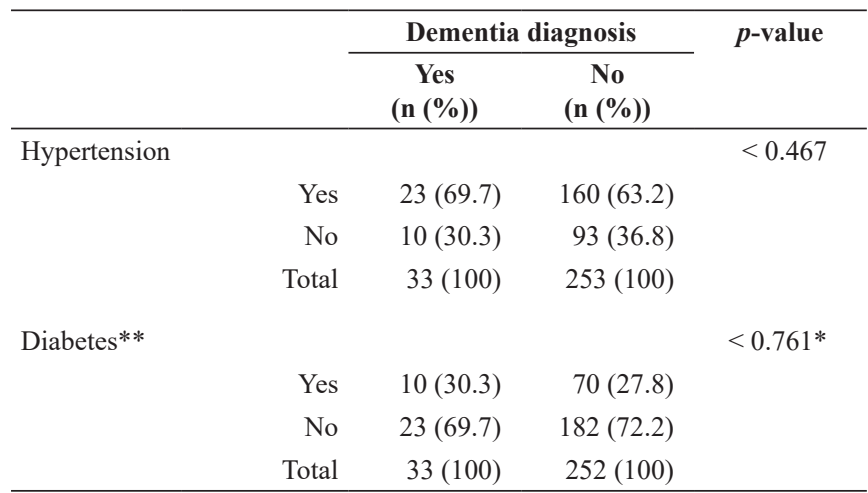

$p$-values based on Chi-square statistics except * where Fisher's Exact Test was used.

** Diabetes data were not available for one control.

Table 9: Dementia pattern/type and hypertension/diabetes

\begin{tabular}{rrrr}
\hline & \multicolumn{2}{c}{ Dementia pattern/type } & p-value \\
\cline { 2 - 3 } & $\begin{array}{c}\text { Alzheimer's } \\
\text { and other } \\
\text { (n (\%)) }\end{array}$ & $\begin{array}{c}\text { Vascular } \\
(\mathbf{n}(\%))\end{array}$ & \\
\hline Both hypertension and diabetes & $6(28.6)$ & $2(16.7)$ & $0.257^{*}$ \\
Hypertension only & $9(42.9)$ & $6(50.0)$ & \\
Diabetes only & $0(0.0)$ & $2(16.7)$ & \\
Neither hypertension nor diabetes & $6(28.6)$ & $2(16.7)$ & \\
All categories & $21(63.6)$ & $12(36.4)$ & \\
\hline
\end{tabular}

* $p$-value based on Fisher's Exact Test. 
the composition of the dementia types by hypertension/ diabetes categories, there was overall no statistically significant relationship among these variables $(p=0.257)$.

\section{DISCUSSION}

This study had combined community investigation with MRI and psychiatric evaluation. It reported that dementia in the Jamaican population was approximately $6 \%$, which was similar to that found in a pilot study by Neita et al (24) and an earlier study by Gao et al (26). The rate was also comparable to the prevalence of $5.7 \%$ and $6.5-8.5 \%$ for the Latin America and Caribbean regions respectively $(4,6)$. As had been reported, more females were affected, supporting the argument for gender-sensitive approaches to prevention and treatment strategies (27). Lower MMSE scores and dementia increased with age, confirming the suggestion made in earlier research that the need for caregiving and support was increasing, especially in the age group of over 80 years, given the ageing of the Jamaican population $(12,27)$.

This study also defined the more common types of deficits seen in dementia, with memory loss, poor executive function and aphasia being the most common deficits. This information is important for designing family and caregiver training and support systems. Being unable to communicate can be an extremely frustrating situation for both the care recipient and the caregiver. Not being able to perform the normal activities of daily living indicates the level of care and support needed. Given the ageing of the Jamaican population, this is an important consideration for the future planning of healthcare and social services.

In addition to important information on prevalence, a critical finding of the study was the level of vascular dementia in those with dementia (32.4\%); this was higher than the rates reported internationally. In addition, almost $50 \%$ of those with Alzheimer's also had significant vascular disease. These findings indicate the importance of recognizing dementia prevention as a public health strategy and strengthening efforts to reduce vascular disease in midlife where vascular disease begins $(28,29)$. Studies have already shown that such efforts can reduce the incidence and impact of dementia $(29,30)$. In light of those observations, we concur with Prince et al (1) who have documented and emphasized the need for both primary and secondary prevention.

Prevention aims to reduce the risk factors which, in the case of vascular dementia, includes hypertension, diabetes, atherosclerosis, lipid abnormalities and smoking and which have been shown to be high in a study of older persons by Eldemire et al and increasing (12). The levels of hypertension were high $(61 \%)$, especially among females (72\%), and had increased (47\%) while diabetes had increased by $157 \%$ since 1989 (12). Given the contribution of hypertension to vascular disease $(8-10)$, this is cause for concern and a reason for urgent action. Increased awareness of the impact of these risk factors beyond the current emphasis on cardiovascular and cerebrovascular diseases to include dementia is warranted.

Both primary and secondary prevention is possible. Primary prevention targets early detection and treatment of vascular factors while secondary prevention targets stroke and diabetes management (30).

\section{CONCLUSION}

The study highlighted dementia as an important consideration for individual practitioners who need to be aware of its possibility in persons with memory loss, not simply attributing the same to ageing. The usefulness of the MMSE as a screening tool to indicate when further investigation is needed has been confirmed, and its potential routine use in primary healthcare settings should be noted by health planners. The latter is particularly important in Jamaica's thrust for enhanced primary healthcare and the strategic development of its health system.

\section{ACKNOWLEDGEMENTS}

This project was supported by a grant from the National Health Fund, Jamaica. Special thanks also go to Elite Diagnostic Imaging Services, Jamaica, and to field staff members C Smallhorne and P Bacchas.

\section{AUTHORS' NOTE}

The authors declare that they have no conflicts of interest.

\section{REFERENCES}

1. Prince M, Bryce R, Albanese E, Wimo A, Ribeiro W, Ferri CP. The global prevalence of dementia: a systematic review and meta-analysis. Alzheimers Dement 2013; 9: 63-75.

2. Baboolal N, Davis G, McRae A. Trinidad and Tobago, a decade of dementia research. Dement Neuropsychol 2014; 8: 330-8.

3. Manes F. The huge burden of dementia in Latin America. Lancet Neurol 2016; 15: 29

4. Prince MJ, Wimo A, Guerchet MM, Ali GC, Wu Y-T, Prina M. World Alzheimer Report 2015 - the global impact of dementia: an analysis of prevalence, incidence, cost and trends. London: Alzheimer's Disease International; 2015. Available from: https://www.alz.co.uk/research/ WorldAlzheimerReport2015.pdf.

5. A neurology revival in Latin America. Lancet Neurol 2015; 14: 1143.

6. Bupa, Alzheimer's Disease International. Dementia in the Americas: current and future cost and prevalence of Alzheimer's disease and other dementias [Internet]. 2017 [cited 2017 Mar 20]. Available from: 
https://www.bupa.com/corporate/our-purpose/aged-care-and-dementia/ reports-and-publications/dementia-in-the-americas\#reportdownload.

7. Statistical Institute of Jamaica. Population Census 2011: Population by Five Year Age Groups by Sex, by Parish. Kingston: Statistical Institute of Jamaica; 2011. Available from: http://statinja.gov.jm/Census/ PopCensus/Populationbyfiveyearsagegroup.aspx.

8. Alagiakrishnan K. Vascular dementia. Medscape [Internet]. Updated 2016 Nov 01; cited 2017 Mar 15. Available from: http://emedicine.medscape.com/article/292105־overview\#a6.

9. Gorelick PB. Risk factors for vascular dementia and Alzheimer disease. Stroke 2004; 35: 2620-2.

10. Grinberg LT, Heinsen H. Toward a pathological definition of vascular dementia. J Neurol Sci 2010; 299: 136-8.

11. Mitchell-Fearon K, Waldron N, James K, Laws H, Holder-Nevins D, Eldemire-Shearer D. Hypertension and diabetes prevalence in older persons in Jamaica, 2012. West Indian Med J 2014; 63: 416-23.

12. Eldemire-Shearer D, James K, Waldron N, Mitchell-Fearon K. Older persons in Jamaica 2012. A report submitted to the National Health Fund, Jamaica. Kingston: Mona Ageing and Wellness Centre, The University of the West Indies; 2014.

13. Hebert LE, Weuve J, Scherr PA, Evans DA. Alzheimer disease in the United States (2010-2050) estimated using the 2010 Census. Neurology 2013; 80: 1778-83.

14. Tombaugh TN, McIntyre NJ. The mini-mental state examination: a comprehensive review. J Am Geriatr Soc 1992; 40: 922-35.

15. Wood RY, Giuliano KK, Bignell CU, Pritham WW. Assessing cognitive ability in research: use of MMSE with minority populations and elderly adults with low education levels. J Gerontol Nurs 2006; 32: 45-54.

16. Grace J, Amick MM. Cognitive screening of older adults. Med Health Rhode Is 2005; 88: 8-11.

17. Folstein M, Anthony JC, Parhad I, Duffy B, Gruenberg EM. The meaning of cognitive impairment in the elderly. J Am Geriatr Soc 1985; 33: $228-35$.

18. Crum RM, Anthony JC, Bassett SS, Folstein MF. Population-based norms for the Mini-Mental State Examination by age and educational level. JAMA 1993; 269: 2386-91.

19. MacKenzie DM, Copp P, Shaw RJ, Goodwin GM. Brief cognitive screening of the elderly: a comparison of the Mini-Mental State
Examination (MMSE), Abbreviated Mental Test (AMT) and Mental Status Questionnaire (MSQ). Psychol Med 1996; 26: 427-30.

20. Folstein MF, Folstein SE, McHugh PR. 'Mini-mental state'. A practical method for grading the cognitive state of patients for the clinician. J Psychiatr Res 1975; 12: 189-98.

21. Pezzotti P, Scalmana S, Mastromattei A, Di Lallo D, Progetto Alzheimer Working Group. The accuracy of the MMSE in detecting cognitive impairment when administered by general practitioners: a prospective observational study. BMC Fam Pract 2008; 9: 29.

22. Cohen-Mansfield J, Marx MS, Thein K, Dakheel-Ali M. The impact of stimuli on affect in persons with dementia. J Clin Psychiatry 2011; 72: 480-6.

23. McHugh ML. Interrater reliability: the kappa statistic. Biochem Med (Zagreb) 2012; 22: 276-82.

24. Neita SM, Abel WD, Eldemire-Shearer D, James K, Gibson RC. The prevalence and associated demographic factors of dementia from a cross-sectional community survey in Kingston, Jamaica. Int J Geriatr Psychiatry 2014; 1: 103-5.

25. Alzheimer's Disease International. World Alzheimer Report 2009. Available from: http://www.alz.co.uk/research/files/ WorldAlzheimerReport.pdf.

26. Gao S, Hendrie HC, Hall KS, Hui S. The relationships between age, sex, and the incidence of dementia and Alzheimer disease: a meta-analysis. Arch Gen Psychiatry 1998; 55: 809-15.

27. Morris C, James K, Eldemire-Shearer D. Gender, culture, retirement, and older men in Jamaica: findings from a survey. CS\&M 2010; 2: $136-53$.

28. O’Donnell CA, Browne S, Pierce M, McConnachie A, Deckers K, van Boxtel MPJ et al. Reducing dementia risk by targeting modifiable risk factors in mid-life: study protocol for the Innovative Midlife Intervention for Dementia Deterrence (In-MINDD) randomized controlled feasibility trial. Pilot and Feasibility Studies 2015 1: 40 .

29. Barnes DE, Yaffe K. The projected effect of risk reduction on Alzheimer's disease prevalence. Lancet Neuro 2011; 10: 819-28.

30. Deckers K, van Boxtel MPJ, Schiepers OJG, de Vugt M, Muñoz Sánchez JL, Anstey KJ et al. Target risk factors for dementia prevention: a systematic review and Delphi consensus study on the evidence for observational studies. Int J Geri Psychiatr 2015; 30: 234-46. 